\title{
La victimización por violencia urbana: niveles y factores asociados en ciudades de América Latina y España
}

\author{
José Miguel Cruz ${ }^{1}$
}

RESUMEN En este artículo se comparan los niveles de victimización por diversas causas y se identifican los factores asociados con ella en ocho ciudades de América Latina y España. Con este propósito se utilizaron los datos del estudio multicéntrico ACTIVA, que se realizó en 1996 bajo la coordinación de la Organización Panamericana de la Salud. La muestra estudiada estuvo constituida por 10821 personas, repartidas entre las ciudades de Salvador de Bahía y Rio de Janeiro, Brasil; Cali, Colombia; Caracas, Venezuela; Madrid, España; San José, Costa Rica; San Salvador, El Salvador, y Santiago, Chile, que representan a la población de cada ciudad entre los 18 y 70 años de edad. Los resultados revelan que los niveles de victimización por diversos tipos de violencia son diferentes en cada ciudad y que las variables asociadas con la victimización con más frecuencia en las ciudades, aunque no en todas, son el sexo, la edad y el consumo de alcohol.

A finales de 1997, uno de los cables informativos de una agencia de noticias rezaba así: "Cuatro personas que dormían en la calle, entre ellas una mujer encinta y dos menores, fueron asesinados esta madrugada en Rio de Janeiro por dos desconocidos que dispararon desde un auto en movimiento, informó la policía" (1). Al año siguiente, otro cable daba cuenta de la siguiente noticia: "Un alto funcionario de la Secretaría de Relaciones Exteriores de México fue asesinado este sábado y su cadáver, con al menos 15 heridas con objeto cortopunzante, fue hallado en la vicealcadía Benito Juárez . . ." (2).

\footnotetext{
Universidad Centroamericana José Simeón Cañas, Instituto Universitario de Opinión Pública, San Salvador, El Salvador. Dirección electrónica: mcruz@iudop.uca.edu.sv, teléfono: (503) 273-4400, ext. 417 , fax (503) 273-7020.
}

Este tipo de noticias inunda los servicios noticieros de la mayoría de los países del continente americano y muestra que la violencia se ha convertido en un mal cotidiano presente en casi todas las sociedades latinoamericanas contemporáneas. Los dos cables anteriores revelan no solo la ubicuidad de la violencia hoy en día -que la hace presente tanto en la ciudad de México como en Rio de Janeiro-, sino que además muestran que la violencia es capaz de generar víctimas de las más distintas características sociales. La violencia que afecta hoy a las ciudades es capaz de convertir en víctimas no solo a los ciudadanos más vulnerables socioeconómicamente, a los más pobres de la Región, sino también a quienes detentan cierto grado de poder.
No es este el espacio para analizar la magnitud de la violencia en cada uno de los países del área, pues se ha tratado extensamente en otros trabajos del estudio sobre la violencia en que se enmarca esta investigación. Baste recordar que América Latina y el Caribe se consideran las zonas más violentas del mundo, ya que en ellas la tasa regional de violencia es de aproximadamente 20 homicidios por 100000 habitantes $(3,4)$. Por otra parte, es posible encontrar diferencias significativas en la magnitud de la violencia entre las ciudades, países y distintas subregiones latinoamericanas. Por ejemplo, países como Colombia y El Salvador han tenido a lo largo de los últimos años tasas que superan las 60 muertes por 100000 habitantes, con alrededor de $25 \%$ de dicha mortalidad atribuible 
CUADRO 1. Tasas de homicidio ( $\times 100000$ habitantes) en algunas ciudades y países de América Latina y España en la década de los noventa. Proyecto ACTIVA, 1998

\begin{tabular}{llr}
\hline \multicolumn{1}{c}{ Ciudad } & Año & Tasa \\
\hline Cali, Colombia $^{a}$ & 1996 & 125,0 \\
Caracas, Venezuela $^{b}$ & 1996 & 56,0 \\
Madrid, España $^{c}$ & 1997 & 3,9 \\
Rio de Janeiro, Brasil $^{d}$ & 1996 & 60,7 \\
São Paulo, Brasile $^{\mathrm{e}}$ & 1993 & 50,2 \\
Costa Rica $^{\mathrm{f}}$ & 1991 & 7,1 \\
San Salvador, El Salvadorg $^{\mathrm{g}}$ & 1996 & 84,5 \\
Santiago, Chile $^{\mathrm{h}}$ & 1996 & 8,0
\end{tabular}

Fuente: Elaboración propia a partir de: a) ref. 5 ; b) ref. 6 ; c) ref. 7 ; d) ref. 8 ; e) ref. $9 ;$ f) ref. 10 ; g) ref. 11; h) ref. 12.

a causas externas, en tanto que en países como Chile y Costa Rica dichas tasas no superan los 10 asesinatos por 100000 habitantes (cuadro 1) $(10,13)$.

\section{La violencia como problema de salud pública}

En la medida en que el problema de la violencia ha ido aumentando, también lo ha hecho la conciencia de su impacto en diversas áreas de la vida social, en especial el área de la salud. La violencia, ante todo, tiene un impacto decisivo en las condiciones de vida de las personas, máxime porque atenta fundamentalmente contra su misma integridad física y su supervivencia (14), al tiempo que menoscaba la calidad de la vida y, a la larga, erosiona las redes básicas de interacción social que sustentan el desarrollo de una comunidad (15). Tal como lo plantea la OPS, la violencia, sobre todo aquella que no concluye con la muerte, altera directamente ese "estado de completo bienestar físico, mental y social" de los afectados que ahora se entiende como salud $(16,17)$. En tal sentido la violencia, en la mayor parte de sus expresiones, se torna productora de enfermedad (18). En ambientes de violencia las personas deben lidiar con condiciones de morbilidad y riesgos de mortalidad que en otras circunstancias no deberían afrontar. La morbilidad por causa de la violencia tiene a su vez un efecto en los sistemas de salud de una sociedad, pues aumenta la de- manda de servicios de salud que muchas veces no están preparados para hacerle frente. La sobrecarga del sistema sanitario no solo se ejerce en la atención de los traumatismos ocasionados por la agresión - que suele ser lo más frecuente-, sino que también involucra otras áreas fundamentales para alcanzar la salud: la rehabilitación física, la atención psicológica, la recuperación de las capacidades productivas y la adaptación a las nuevas limitaciones orgánicas.

En vista de la situación, en la XXXVII Reunión del Consejo Directivo de la OPS se decidió declarar a las conductas violentas como un problema de salud pública y se instó a los distintos Gobiernos de la Región a establecer políticas y planes nacionales y a movilizar recursos para la prevención y el control de la violencia, haciendo especial hincapié en los grupos más vulnerables (9). Lo anterior implica definir el problema y conocer, por consiguiente, cuáles son esos grupos más vulnerables.

\section{Violencia y violencia urbana: definiciones}

El concepto de violencia tiene varias acepciones, si bien en su sentido más genérico se refiere al uso de la fuerza extrema. Sin embargo, la definición adoptada para entenderla como problema de salud pública se refiere al "uso o amenaza de uso de la fuerza física con la intención de hacer daño a otro o hacerse daño" (18). En esta defi- nición hay dos elementos que es importante destacar. En primer lugar, el concepto se refiere a la fuerza física y en tal sentido se excluyen otros tipos de agresiones que no se enmarcan en el ámbito de lo corporal. En segundo lugar, el concepto incluye también la variable de la intencionalidad, la cual excluye aquellos hechos no intencionados que producen lesiones, es decir, los accidentes. Este fue el concepto adoptado por los investigadores del estudio multicéntrico ACTIVA y el que guió el examen de la violencia y de las actitudes y normas que la sustentan (19). Pero al hablar de la violencia urbana se debe hacer una precisión adicional. Muchos autores se refieren al término violencia urbana aproximándolo a criminalidad $(20,21)$; aunque la mayor parte de la violencia ejercida en contra de una persona tiene una dimensión delictiva y, por tanto, está penada socialmente, usualmente se ha adoptado el término de violencia urbana para hacer referencia al crimen cometido en los entornos públicos de las grandes ciudades. Así, la violencia urbana sería aquella ejercida en el marco de las relaciones y dinámicas mediadas por la convivencia urbana, cuyas expresiones más frecuentes son el robo a mano armada, las amenazas, las agresiones, los golpes, los secuestros y el homicidio.

\section{Victimización}

Una vez definida la violencia urbana, la victimización sería el acto en el cual una persona es objeto del uso de la fuerza, que le produce un daño físico o psicológico. ¿Quiénes suelen ser las víctimas de la violencia urbana? En el pasado, según algunos compendios de criminología, se solía abordar el problema de las víctimas, sobre todo el de la propensión a ser víctima (victimproneness), por medio del examen de las "debilidades" biológicas o situacionales que convertían en víctima a una persona $(22,23)$. Según esta perspectiva, por consiguiente, las víctimas poseían características personales que, a diferencia del victimario, las hacían vulnerables (ser mujer, joven, anciano, 
retrasado mental, etc.). En la actualidad, los enfoques adoptados en el examen de la victimización muestran, antes que diferencias, la proximidad de las características demográficas de las víctimas y los victimarios (24). Así, desde la perspectiva de la salud pública, importa más saber quiénes suelen ser las víctimas más frecuentes desde el punto de vista demográfico y conductual que según categorías de la personalidad. En ese sentido, la mayor parte de los estudios revelan que la victimización por la violencia, sobre todo la que termina con la muerte o con el daño físico de la persona, se encuentra asociada con ciertas variables demográficas. De hecho, en los Estados Unidos de América, de donde provienen la mayor parte de los estudios sobre el tema, la edad constituye la variable más importante en la predicción del riesgo de victimización violenta $(24,25)$. Otras variables que aparecen asociadas con la victimización por la violencia urbana, según algunos de los estudios, son el sexo, el nivel socioeconómico de la víctima o de la comunidad donde reside la víctima y la raza o grupo étnico de esta. De acuerdo con la Encuesta Nacional de Victimización por Crimen de 1994 de los Estados Unidos, "los hombres, las personas de color, los hispanos, los jóvenes, los pobres y los habitantes del centro de las ciudades fueron los más vulnerables a la violencia" (26).

En América Latina, las tendencias de algunas variables parecen ser muy parecidas. En una revisión por Yunes (27) de las tasas de mortalidad por causas violentas durante la década de los ochenta se encontró que en todos los países estudiados de América Latina y el Caribe la razón entre las tasas de mortalidad en hombres y mujeres fue siempre mayor de 1 y en todos - con la excepción de Cuba- la diferencia fue mayor que la observada en los Estados Unidos. Una investigación realizada en Rio de Janeiro reveló que las víctimas más frecuentes de los delitos de robo, agresión y extorsión entre 1995 y 1996 fueron las personas con menos de 30 años de edad y de ellas, las de sexo masculino (28). En Caracas, Sanjuán constató que aproximada- mente $95 \%$ de las víctimas de homicidio eran hombres y que $70 \%$ pertenecían al grupo de edad entre los 15 y 29 años (6); mientras que en Cali 53\% de los homicidios registrados tuvieron como víctima a un hombre de 15 a 29 años de edad (29). En San Salvador, un hombre entre los 16 y 25 años de edad tenía una probabilidad de morir asesinado 10 veces mayor que la de una mujer del mismo grupo de edad (30). Se podrían seguir citando estudios y revisiones de los registros de distintas ciudades de la Región, pero en la mayoría de los centros urbanos latinoamericanos las tendencias respecto a la edad y el sexo son similares (31).

En América Latina, los estudios publicados no son concluyentes respecto a otras variables personales que pueden estar asociadas con la victimización por la violencia urbana. Aunque pertenecer a un sector socioeconómico bajo suele considerarse un factor de riesgo de victimización violenta, algunos estudios sugieren que la condición personal de la víctima pesa más que el contexto ambiental donde el acto violento se produce. Por ejemplo, Akerman (32) encontró que en São Paulo los robos predominan en áreas donde son mejores las condiciones de vida y, en cambio, los homicidios ocurren en áreas periféricas de baja extracción económica. Por otro lado, en un estudio realizado en Santiago, Chile, se observó que los "robos con fuerza" se encuentran parcialmente concentrados en áreas de estratos medios y altos, mientras que los "robos con violencia" -que suponen un ejercicio más severo de la violencia y que afectan a las personas - suelen ocurrir en barrios de clase media y baja (33).

En el orden conductual existen otras variables, como el consumo de alcohol y la tenencia de armas, que parecen estar asociadas con la victimización. Por lo tanto, su presencia se convierte en un factor de riesgo de sufrir una agresión. Por lo general, estas variables se han asociado más con el perpetrador de la violencia que con la víctima $(24,25)$. Empero, el análisis del problema de la victimización desde otro prisma ha permitido encontrar también ciertas conexiones entre las víctimas, el consumo de alcohol y la tenencia de armas. En Cali se encontró que $25 \%$ de las víctimas de asesinato estaban intoxicadas por alcohol (34), mientras que en El Salvador, el porcentaje de las víctimas de violencia hospitalizadas por intoxicación alcohólica ascendió de 20 en una semana normal a 35 en una semana festiva (35). En el caso de la tenencia de armas, Guerrero sostiene, citando un estudio estadounidense (34), que la posesión de un arma de fuego se asocia con un riesgo de morir 2,7 veces mayor para los integrantes del hogar.

En este campo de la victimización por la violencia urbana, el presente estudio se propone revisar los resultados regionales del estudio multicéntrico ACTIVA. Con ese fin en este artículo se realizará, en primer lugar, una comparación de las frecuencias de la victimización por robo, amenazas, agresión y heridas en las ocho ciudades que participaron en el estudio, buscando establecer semejanzas y diferencias entre ellas. En segundo lugar, se intentará constatar cuáles son las variables demográficas y conductuales que están asociadas con la victimización por la violencia y las características más frecuentes de las víctimas en cada una de las ciudades participantes.

\section{MATERIALES Y MÉTODOS}

La descripción de la muestra del estudio ACTIVA se ha hecho en otro artículo dedicado al diseño metodológico del estudio regional (véase la pp. 222-231). Baste entonces decir, para los propósitos de este apartado, que el estudio ACTIVA se basó en una muestra de 10821 entrevistas repartidas entre ocho ciudades: Salvador de Bahía, Brasil $(n=1384)$; Cali, Colombia $(n=2288)$; Caracas, Venezuela ( $n=$ $1297) ;$ Madrid, España $(n=1105) ;$ Rio de Janeiro, Brasil $(n=1114)$; San José, Costa Rica $(n=1131)$; San Salvador, El Salvador $(n=1290)$, y Santiago, Chile $(n=1212)$. La muestra se estratificó por conglomerados según el nivel socioeconómico y la densidad de la población. Las personas entrevistadas se seleccionaron en cada hogar por 
medio de un muestreo sistemático sin sustitución. Se supone que la muestra de cada ciudad sea representativa de la población entre los 18 y 70 años de edad. En el muestreo se pretendió realizar estimaciones con una confianza del 95\%.

Para los propósitos de los análisis incluidos en este artículo se utilizó la parte del cuestionario establecido por el proyecto ACTIVA. En las ocho ciudades incluidas en la muestra regional se administró un cuestionario integrado por 14 preguntas que recogían diversos tipos de victimización a causa de la violencia criminal. En el presente trabajo se utilizaron variables a partir de algunos ítemes y escalas del instrumento. En algunos casos, estas variables corresponden directamente a los resultados de ítemes individuales; en otros casos, sobre todo los que se refieren a la victimización, surgen del tratamiento de varios ítemes. Algunas de estas variables fueron construidas por Orpinas durante el trabajo de compilación y depuración de las bases de datos del proyecto ACTIVA (36), mientras que otras fueron desarrolladas por el autor. Asimismo, se omitieron los ítemes que reflejan la observación de victimización y los referidos a homicidios y suicidios, los primeros porque no se refieren a la victimización directa del entrevistado y los segundos, a pesar de su importancia en la definición de la violencia urbana, porque la formulación del reactivo impedía recoger las características propias de la víctima, que es lo esencial para este trabajo. En todo caso, hay que subrayar que el instrumento - dado el carácter del estudio basado en entrevistas- recoge declaraciones sobre las experiencias de victimización y no registra los hechos en sí mismos; por tanto, las variables se refieren a la notificación del suceso y dependen de las declaraciones de los encuestados en cada uno de los países.

Las variables demográficas analizadas corresponden a los reactivos del cuestionario que recogían información sobre el sexo, edad y estrato socioeconómico del entrevistado. Los valores de la variable edad se recategorizaron para hacerlos más manejables en el análisis. Los entrevistados se dividieron en cuatro grupos de edad, tomando en cuenta la distribución de las tendencias de victimización por la violencia: de 18 a 25 años, de 26 a 35, de 36 a 50 , y de 51 a 70 . Los valores de la variable estrato socioeconómico dividen a la población de cada una de las ciudades en las siguientes categorías: alto, medio y bajo.

Las variables conductuales fueron: a) frecuencia del consumo de alcohol. Esta es una variable ordinal que recoge el consumo de cierta cantidad de alcohol en el lapso de un mes: ¿Cuántas veces en el último mes ha tomado más de cinco tragos de licor o botellas (latas) de cerveza en una sola ocasión? Los valores de la respuesta se expresaron en: nunca, una vez, tres o cuatro veces, cinco a 10 veces, y más de 10 veces. b) Tenencia de armas. Variable dicotómica que recoge la posesión de armas de fuego por parte del entrevistado: ¿Tiene usted algún arma de fuego (pistola, revólver, rifle) en su casa?

Las variables de victimización fueron las siguientes. a) Víctima de robo a mano armada. Variable dicotómica generada a partir del siguiente ítem: ¿Alguien le robó algo a mano armada en los últimos 12 meses? Sus valores denotan la condición de haber sido o no víctima al menos una vez de algún hecho de ese tipo. b) Víctima de amenazas. Variable construida a partir de tres reactivos: ¿En los últimos 12 meses, algún policía u otra autoridad pública le exigió dinero?, ¿Alguien que no era un policía o agente de la seguridad pública le amenazó para sacarle o pedirle dinero en los últimos 12 meses? y ¿En los últimos 12 meses le amenazaron para forzarle a cambiar su lugar de residencia, cambiar sus opiniones o quedarse callado/a respecto a algo que usted conoce?, que expresa la victimización o no, al menos una vez, por amenazas. c) Víctimas de agresiones. Variable dicotómica construida a partir del siguiente ítem: ¿Fue usted golpeado por otra $u$ otras personas en los últimos 12 meses? Esta variable expresa la condición de haber sido víctima por lo menos una vez de una agresión a golpes por parte de un particular. d) Víctimas de heridas. Variable dicotómica construida a partir de los siguientes reactivos: ¿Fue usted herido con un arma blanca en los últimos 12 meses? y ¿Fue usted herido con un arma de fuego en los últimos 12 meses? Esta expresa la condición de haber sido herido o no, por lo menos una vez, por arma de fuego o arma blanca. e) Victimización global. Variable que reúne en sí misma todas las victimizaciones anteriores (robo, amenazas, agresiones y heridas). Dado que cada una de las victimizaciones incluidas en esta variable son diferentes en cuanto al impacto para la persona que sufre el hecho, cada tipo de victimización se ponderó empíricamente a partir de una cantidad inversamente proporcional a la frecuencia de cada uno de los hechos, es decir, la victimización más frecuente (robo) tendría un valor igual a $1 \mathrm{y}$, en la medida en que disminuye la frecuencia de un tipo de victimización, su peso aumenta (por ejemplo, heridas $=15$ ). Con ello se evita atribuir un mismo nivel de afectación a acontecimientos tan disímiles como las amenazas y las heridas. Ahora bien, esta variable de victimización global se utilizó en dos expresiones distintas. En la primera fase del análisis se utilizó en forma dicotómica, donde un valor expresaba al menos una experiencia de victimización y el otro, la ausencia de victimización. En la segunda parte del análisis se utilizó la misma variable de forma continua y normalizada mediante su transformación a escala logarítmica en base de 10 . Esta variable guardó una correlación aceptable con la variable continua original ( $r$ de Pearson $=0,7810$ ).

La descripción de los métodos de recolección de datos de cada una de las ciudades se presenta en el artículo sobre el diseño metodológico de ACTIVA. Aquí solo es necesario subrayar que en todas las ciudades las entrevistas se realizaron de forma personal y mediante la visita al hogar del entrevistado. Todas las entrevistas fueron codificadas y los resultados se registraron en una base de datos informatizada en la ciudad donde se hizo la encuesta. Por tanto, las bases de datos fueron locales. Se revisaron localmente y posteriormente se enviaron al centro de acopio regional, donde fueron sometidas a otra depuración y compila- 
das en una sola base de formato SPSS para Windows (36). Los datos utilizados en este trabajo fueron analizados usando el mismo programa (37)

Dos partes conforman el procedimiento de análisis de estos datos. En primer lugar, se llevó a cabo una comparación general de las distintas clases de victimización por ciudad, tratando de establecer la magnitud de los diversos tipos de violencia que han sufrido los ciudadanos en cada una de las ocho ciudades del estudio. Para ello, se examinaron los porcentajes de victimización de cada hecho violento estudiado por ciudad y se calcularon los porcentajes de los niveles de victimización por violencia que afrontan los habitantes de cada una de las ciudades incluidas en el estudio. En segundo lugar (lo que constituye la parte medular de este trabajo), se averiguó la posible existencia de asociaciones entre los factores demográficos y conductuales y la variable continua de victimización global en cada una de las ciudades. Para ello se realizaron análisis de la varianza. Cuando esto no fue posible, porque las varianzas entre los grupos fueron heterogéneas, se usó una prueba $t$ de Student para varianzas no homogéneas $(37,38)$. Cuando se compararon más de dos grupos, se construyeron análisis de la varianza de Kruskal-Wallis (39). Para evitar el problema de las comparaciones múltiples se aplicó la prueba de Scheffé.

El criterio para utilizar la variable de victimización global y no las de agresión y heridas - que tienen mayor relevancia en salud pública - fue que el bajo número de casos de victimización por agresión y heridas en cada ciudad no permitía realizar un análisis estadístico robusto. La variable de victimización global tiene la ventaja de incorporar un buen número de casos por ciudad, además de que, por medio de la ponderación, otorga el peso debido a los hechos de victimización más graves y permite establecer en qué medida la gente ha sido victimizada por la violencia urbana según sus propias declaraciones. Por esa razón, en la segunda parte del análisis se decidió utilizar la variable de victimización global en forma de índice y no como porcentajes de valores dicotómicos, para así poder considerar dentro de la relación el peso de las victimizaciones más graves (agresiones y heridas) sin menoscabar la validez del análisis.

\section{RESULTADOS}

\section{Tipos de victimización por violencia urbana en las ciudades}

En la figura 1 aparecen los porcentajes de victimización por las distintas causas estudiadas. Mientras que en San Salvador el porcentaje de personas que notificaron haber sufrido algún tipo de violencia ascendió a 38,5\%, en Santiago esta cifra no llegó a $11 \%$. Se observa, además, que aparte de San Salvador, en otras ciudades como Caracas, Salvador de Bahía y Cali se registraron altos niveles de victimización global $(30,6 \%, 29 \%$ y $27,4 \%$, respectivamente), en tanto que en el resto de las ciudades los porcentajes fueron más bajos: Rio de Janeiro, 17\%; San José, 15\%; y Madrid 13,7\%.

De acuerdo con las declaraciones de los entrevistados, en la mayoría de las ciudades, a excepción de Madrid y San Salvador, el robo a mano armada fue el tipo de victimización más frecuente; en Salvador de Bahía se registró el por- centaje más alto $(22,1 \%)$ y en Santiago de Chile, el más bajo (6,9\%). Las amenazas de agresión o muerte y los actos de extorsión produjeron en San Salvador un elevado porcentaje de víctimas (cercano a 25\%); en cambio, en Santiago y en Salvador de Bahía esta cifra no superó $6 \%$. Los porcentajes de acciones violentas más graves, como las agresiones y las heridas (por arma de fuego o arma blanca), fueron los más bajos. En Cali, Salvador de Bahía y Rio de Janeiro el porcentaje de las víctimas por agresión o golpes fue mayor de $5 \%$ (en Cali, el porcentaje ascendió a $7,2 \%)$, mientras que en Madrid y Santiago no pasó de 2,6\%. Finalmente, la victimización por heridas fue más frecuente en Cali, en tanto que en las ciudades de Madrid, San José, Santiago y Caracas el porcentaje de heridos no alcanzó siquiera $1 \%$.

El análisis de la varianza no reveló diferencias estadísticamente significativas entre los niveles de victimización global de las ciudades de Madrid, San José y Santiago $(P>0,05)$, ni entre las de Salvador de Bahía, Cali y Caracas. Tampoco se detectaron diferencias entre Rio de Janeiro, Madrid y San José. Por el contrario, las diferencias fueron significativas entre San Salvador y todas las ciudades restantes $(P<0,05)$.

FIGURA 1. Porcentajes de victimización por distintas causas según ciudad. Proyecto ACTIVA, 1998

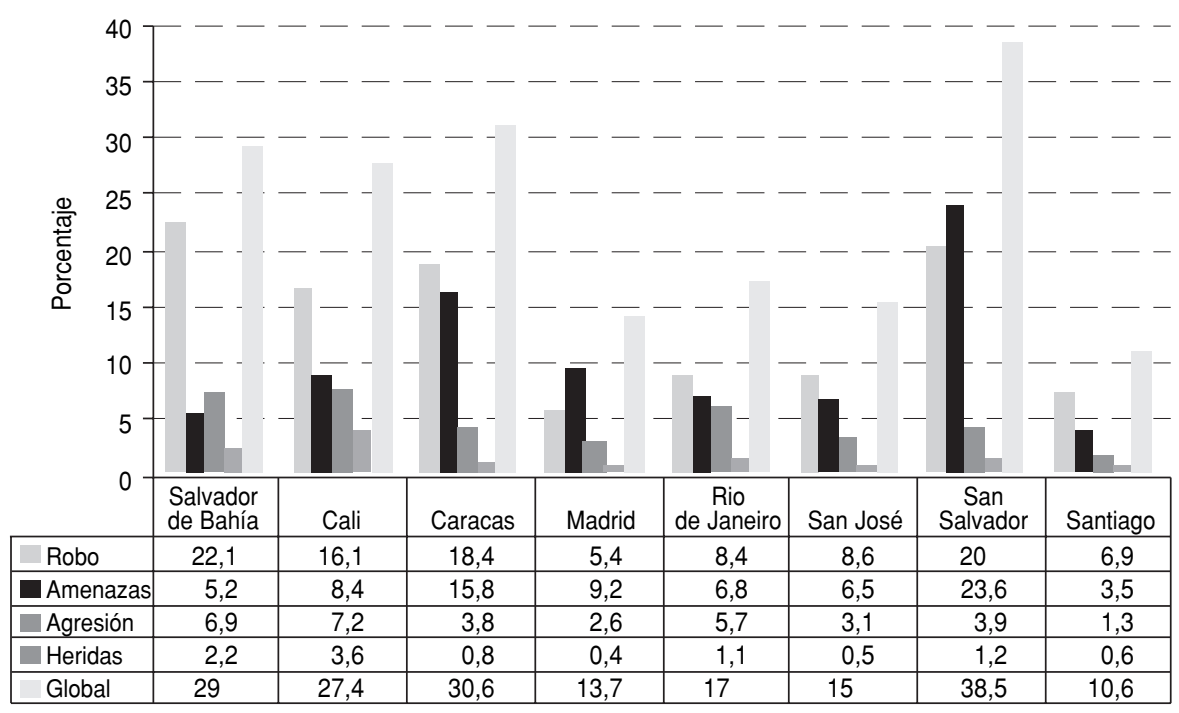




\section{Variables asociadas con la victimización urbana}

En el cuadro 2 figuran los índices de victimización global para cada una de las ciudades estudiadas según el sexo, la edad, el estrato socioeconómico, el consumo de alcohol y la tenencia de armas por parte de la víctima. Como puede observarse, los índices de victimización global por violencia no variaron siempre de la misma forma en todas las ciudades. En Salvador de Bahía, el sexo de la víctima y su consumo de alcohol constituyeron las variables discriminadoras de los índices de victimización $(P<0,05)$. En los hombres bahianos se detectó un nivel de victimización más alto que en las mujeres, en tanto que aquellos que suelen consumir alcohol con más frecuencia sufrieron más actos violentos. Aunque en el caso de la edad es posible advertir un índice más alto en el grupo de 18 a 25 años, no se encontraron diferencias estadísticamente significativas entre la edad ni entre el resto de las variables incluidas en el análisis. En el caso de Cali, las víctimas más frecuentes de la violencia general fueron los hombres más jóvenes, que consumen alcohol con más frecuencia y que suelen poseer un arma de fuego. Asimismo, los índices de victimización fueron algo más elevados en el estrato socioeconómico alto, si bien las diferencias observadas no fueron estadísticamente significativas $(P>0,05)$. En Caracas, todas las diferencias entre las variables estudiadas fueron estadísticamente significativas: el sexo de la víctima (los hombres más que las mujeres); la edad (los jóvenes entre 18 y 25 años más que los de cualquier otro grupo de edad); el estrato socioeconómico (las personas de estrato bajo en mayor medida que las de los otros estratos); el consumo frecuente de alcohol, así como la tenencia de armas. Los índices de victimización global en Madrid solo variaron significativamente en cuanto a edad y consumo de alcohol. En el primer caso, el índice más elevado también se encontró entre los jóvenes, mientras que en el segundo los que consumían más alcohol habían sufrido más acciones derivadas de la violencia urbana. En Rio de Janeiro, los índices de victimización fueron más elevados y estadísticamente distintos del resto en los hombres, las personas más jóvenes y los que consumen alcohol con más frecuencia. En San José, los hombres, los jóvenes, los que ingieren alcohol más frecuentemente y los que tienen un arma de fuego fue-

CUADRO 2. Índice de victimización global (0-2,5) por ciudad según variables personales y conductuales. Proyecto ACTIVA, 1998

\begin{tabular}{|c|c|c|c|c|c|c|c|c|}
\hline Variable & \multicolumn{8}{|c|}{ Ciudad } \\
\hline \multicolumn{9}{|l|}{ Sexo } \\
\hline Masculino & 0,180 & 0,236 & 0,256 & 0,078 & 0,154 & 0,122 & 0,337 & 0,055 \\
\hline Femenino & 0,135 & 0,125 & 0,122 & 0,068 & 0,072 & 0,045 & 0,281 & 0,054 \\
\hline $18-25$ & 0,180 & 0,234 & 0,230 & 0,113 & 0,168 & 0,125 & 0,365 & 0,069 \\
\hline $26-35$ & 0,153 & 0,177 & 0,184 & 0,078 & 0,108 & 0,075 & 0,339 & 0,052 \\
\hline $36-50$ & 0,158 & 0,144 & 0,162 & 0,052 & 0,109 & 0,070 & 0,289 & 0,052 \\
\hline \multirow[t]{2}{*}{$51-70$} & 0,120 & 0,134 & 0,108 & 0,050 & 0,046 & 0,048 & 0,201 & 0,043 \\
\hline & $F=1,79$ & $F=9,24^{a}$ & $F=7,51^{a}$ & $F=5,57^{a}$ & $F=7,41^{a}$ & $F=5,89^{a}$ & $F=6,15^{a}$ & $F=0,87$ \\
\hline \multicolumn{9}{|l|}{ Estrato } \\
\hline Nunca & 0,123 & 0,134 & 0,135 & 0,063 & 0,086 & 0,066 & 0,290 & 0,048 \\
\hline 1 ó 2 & 0,173 & 0,206 & 0,195 & 0,081 & 0,135 & 0,115 & 0,362 & 0,046 \\
\hline 3 ó 4 & 0,202 & 0,304 & 0,201 & 0,088 & 0,204 & 0,101 & 0,392 & 0,131 \\
\hline 5 a 10 & 0,242 & 0,317 & 0,300 & 0,187 & 0,099 & 0,164 & 0,371 & 0,085 \\
\hline \multirow{2}{*}{ Más de 10} & 0,204 & 0,583 & 0,328 & 0,193 & 0,277 & 0,474 & 0,455 & 0,030 \\
\hline & $H=21,41^{\mathrm{a}}$ & $H=105,1^{\mathrm{a}}$ & $H=18,36^{a}$ & $H=12,32^{\mathrm{a}}$ & $H=23,14^{\mathrm{a}}$ & $H=19,75^{a}$ & $H=8,60$ & $H=14,11^{a}$ \\
\hline \multicolumn{9}{|l|}{ Armas } \\
\hline Sí & 0,226 & 0,304 & 0,252 & 0,070 & 0,127 & 0,124 & 0,333 & 0,081 \\
\hline \multirow{2}{*}{ No } & 0,152 & 0,169 & 0,166 & 0,072 & 0,105 & 0,072 & 0,304 & 0,052 \\
\hline & $t=1,80$ & $t=3,19^{a}$ & $t=2,46^{\mathrm{a}}$ & $F=0,00$ & $F=0,28$ & $t=2,20^{\mathrm{a}}$ & $F=0,29$ & $F=2,44$ \\
\hline
\end{tabular}

$\bar{a} P<0,05$. 
ron los más perjudicados por la violencia. Los resultados del análisis estadístico señalan que en San Salvador las diferencias en los índices de victimización relativas al sexo, la edad y el estrato socioeconómico fueron estadísticamente significativas, y los índices más altos en cada una de esas variables indican una historia más frecuente de victimización que en el resto. Finalmente, en Santiago, Chile, la única variable asociada estadísticamente con el índice de victimización general por violencia fue el consumo de alcohol: en las personas que consumían alcohol de tres a cuatro veces al mes se registró el mayor nivel de victimización.

\section{DISCUSIÓN}

La comparación entre los niveles de victimización de las ocho ciudades estudiadas en el proyecto ACTIVA pone de manifiesto, en primer lugar, que las tendencias de victimización registradas en el estudio multicéntrico muestran cierta similitud con las tendencias mostradas por las tasas de homicidios en el cuadro 1 como indicadores de violencia locales sobre los cuales se dispone de información. Esto sugiere que detrás de esos niveles de violencia expresados formalmente por las tasas de homicidio también se encuentran otras formas de victimización que forman parte del problema de la violencia urbana y que afectan a cada una de las ciudades, y dentro de las cuales el homicidio sería su expresión más palmaria. La comparación de los porcentajes de victimización de las ciudades estudiadas revela que en Santiago, San José y Madrid se registran, al igual que con sus tasas de homicidios, los niveles más bajos de victimización por violencia, a diferencia de ciudades como San Salvador, Cali y Caracas, en las cuales los dos sistemas de registro empleados - ACTIVA y tasas de homicidios- permitieron detectar altos índices de victimización. Esto no debe significar que la variable de victimización de ACTIVA se construyera con el propósito de que remedara las tasas de homicidios; solamente se pretende se- ñalar la utilidad de medidas como esta para aproximarse al problema de la violencia y mostrar las distintas dimensiones en que puede expresarse.

En segundo lugar, la comparación muestra que los niveles de victimización - expresados como porcentajes de personas que dicen haber sufrido algún acto de violencia- entre las ciudades no solo son muy distintos en cuanto a su magnitud, sino también en lo que se refiere a la tipología de la victimización. En otras palabras, los actos que en una ciudad se conforman como el generador más común de víctimas por la violencia, en otra ciudad apenas figuran como causa de victimización. Por ejemplo, los datos muestran que los residentes de San Salvador son víctimas de la violencia general en una proporción dos veces más alta que la de los residentes de Rio de Janeiro; sin embargo, estos últimos sufren agresiones en un porcentaje mayor que los habitantes de aquella ciudad centroamericana. Particular atención debe prestarse a los niveles de agresión y lesión por heridas detectados en Cali y Bahía y a los niveles de agresión de Rio de Janeiro, que duplican o triplican los de cualquier otra ciudad incluida en el estudio multicéntrico y muestran el impacto sustancial que ejercen en la victimización global de esas ciudades. A juzgar por las declaraciones de los entrevistados en Salvador de Bahía, Caracas, San Salvador y Cali, los ciudadanos están expuestos al robo a mano armada en una proporción dos veces mayor que en las ciudades de Madrid, Rio de Janeiro, San José y Santiago.

Estos datos subrayan un hecho importante constatado en este estudio: la violencia urbana es diferente en cada sitio, de tal forma que, a pesar de que Cali y San Salvador tienen niveles muy altos de victimización por violencia urbana, ello no significa que la configuración y el impacto de esa victimización sean los mismos para ambas ciudades. En San Salvador, por ejemplo, parece prevalecer un tipo de victimización basado en hechos que pueden ser enmarcados en la delincuencia callejera: robos y amenazas; en cambio, aunque en Cali se registró una proporción elevada de robos, destaca, al igual que en Bahía, el alto porcentaje de victimización por hechos violentos más graves: agresiones y heridas. $\mathrm{Al}$ cabo, ambas ciudades se encuentran entre las más violentas de la Región, si bien estas diferencias pueden explicar que los generadores y receptores de la violencia no sean las mismas personas. Esto confirma el supuesto de que no es lo mismo afrontar la violencia en Cali que en San Salvador, y de que cualquier estrategia de atención del problema y sus consecuencias en la salud debe tener en cuenta las diferencias consignadas para hacer más efectivas las tareas de prevención.

Cabe recordar, por otro lado, que como estos niveles de victimización dependen de las declaraciones que hacen las personas, el registro de las victimizaciones notificadas en el estudio puede estar sujeto a errores de la memoria de los entrevistados. Dado el carácter grave de ciertos acontecimientos como las heridas y a pesar del esfuerzo de los investigadores locales por dejar claro el marco temporal en las entrevistas, algunos entrevistados podrían haber sobrenotificado el acontecimiento de los hechos violentos. En tal sentido se estaría sobreestimando la ocurrencia de los mismos. Aunque no es posible confirmarlo, esta posible sobreestimación ha de considerarse una limitación consustancial al método de recolección de información por encuestas.

En cuanto a las variables asociadas con el fenómeno de la victimización por hechos violentos cabe destacar que la posibilidad de que una característica personal se convierta en un factor de riesgo de victimización depende también del lugar - de la ciudad-en que habita la persona y, por tanto, del tipo de violencia que prevalezca en esa zona. Esta afirmación se basa en que el estudio no arrojó pruebas concluyentes de que una variable concreta (por ejemplo, la edad), a pesar de su importancia reconocida, constituya un factor diferencial en todas las ciudades estudiadas. Los resultados del estudio multicéntrico indican que hay variables, o características personales, que se relacionaron con la victimización en la mayor parte de las ciudades, aun- 
que no en todas: el sexo, la edad y el consumo de alcohol. En seis de las ocho ciudades (Salvador de Bahía, Cali, Caracas, Rio de Janeiro, San José y San Salvador) las víctimas más frecuentes de la violencia urbana resultaron ser los hombres. Sin embargo, esto no quiere decir que en las otras ciudades (Madrid y Santiago) las mujeres sean quienes sufran más violencia; únicamente significa que los datos disponibles no ofrecen pruebas que respalden la noción de que en las capitales de España y Chile el sexo constituya un criterio para diferenciar a las víctimas de la violencia. Esto podría deberse a que en lugares donde los niveles de afectación por violencia son muy bajos, las diferencias entre víctimas en cuanto al sexo no llegan a ser muy grandes $\mathrm{y}$, por tanto, la baja probabilidad de sufrir una agresión es casi igual para hombres y mujeres.

Por lo que respecta a la edad, los resultados del proyecto ACTIVA confirman en seis ciudades las tendencias reveladas por otros estudios: las personas más jóvenes —entre los 18 y los 25 años de edad - resultaron ser el grupo más victimizado a causa de la violencia. Esto es cierto para los habitantes de Cali, Caracas, Madrid, Rio de Janeiro, San José y San Salvador, pero no para los de Salvador de Bahía y Santiago. No obstante, el hallazgo de una asociación entre edad y victimización no se limita al grupo de los más jóvenes; en todas las ciudades en que se encontró una relación entre la edad y la victimización se observó que a medida que aumenta la edad, disminuye el índice de victimización, de tal manera que la probabilidad de ser víctima de un acto violento desciende con la edad de la víctima.

Por otro lado, en todas las ciudades, con excepción de San Salvador, las personas que consumían alcohol con más frecuencia notificaron ser víctimas con mayor asiduidad. Más aún, en la mayor parte de las ciudades el índice de victimización corrió paralelo con la frecuencia del consumo de alcohol; es decir, a mayor consumo de alcohol, mayor índice de victimización. Estos resultados sustentan la tesis según la cual el alcohol no solo constituye un factor asociado con el ejercicio de la violencia, sino también con las posibilidades de ser víctima de una acción violenta. Como el consumo de alcohol es una variable conductual y, por tanto, sujeta a modificación, este hallazgo tiene implicaciones para las actividades preventivas que puedan emprenderse con objeto de paliar el problema.

En cuanto a las otras variables estudiadas, solo se encontraron asociaciones entre el estrato socioeconómico y la tenencia de armas de fuego en algunas ciudades. En Caracas y San Salvador la violencia urbana se observó más frecuentemente en los estratos bajos que en los restantes, mientras que en Cali, Caracas y San José la tenencia de armas, además de ser muy frecuente, constituyó un factor asociado con la victimización.

Para concluir, este trabajo revela que la victimización por actos de violencia notificada por las personas varía en la mayor parte de las ciudades estudiadas. Estas diferencias no se deben atribuir solamente a los niveles de violencia, que pueden ser muy diferentes, sino también a las expresiones de la violencia que afectan de manera muy diversa a las personas. Las diferencias en la magnitud de la violencia y en sus expresiones podrían estar relacionadas, hasta cierto punto, con las características de las víctimas más frecuentes de esa violencia. No obstante, los resultados indican que la edad, el sexo y el consumo de alcohol son las variables que están más claramente asociadas con la victimización: los hombres, las personas de menor edad y las que consumen alcohol constituyen los grupos que padecen agresiones con mayor frecuencia en la mayoría de las ciudades estudiadas, al tiempo que definen los grupos de riesgo que precisan la vigilancia más constante en la lucha contra la violencia urbana.

Agradecimiento. Este artículo se ha basado en los datos del Estudio Multicéntrico sobre Actitudes y Normas Culturales frente a la Violencia (proyecto ACTIVA), que se realizó en ocho ciudades de América Latina y España bajo el auspicio y la coordinación de la Organización Panamericana de la Salud (OPS/OMS). El autor desea agradecer a Rubí Esmeralda Arana, Giovanni Flores y David Navarro su colaboración en la preparación de su contenido.

\section{REFERENCIAS}

1. Agencia de noticias EFE. Asesinan a cuatro indigentes. San Salvador: El Diario de Hoy, 10 de diciembre de 1997:22.

2. Agencia de noticias EFE. De quince heridas con arma blanca ultiman alto funcionario mexicano. San Salvador: La Prensa Gráfica, 10 de agosto de 1998:50.

3. Banco Mundial. El crimen y la violencia como problemas para el desarrollo en América Latina y el Caribe. Presentado en el seminario El Desafío de la Criminalidad Urbana. Rio de Janeiro: Banco Interamericano de Desarrollo; 1997.
4. Bobadilla JL, Cárdenas V, Couttolenc B, Guerrero R, Remenyi MA. Medición de los costos de la violencia. Resultados de un taller organizado por la Organización Panamericana de la Salud (OPS) y el Banco Interamericano de Desarrollo. Caracas: OPS; 1995.

5. Rubio M. Criminalidad urbana en Colombia. Presentado en el seminario El Desafío de la Criminalidad Urbana. Rio de Janeiro: Banco Interamericano de Desarrollo; 1997.

6. Sanjuán AM. La criminalidad en Caracas: percepciones y realidades. Rev Venezolana Econ Ciencias Soc 1997;3:215-254.
7. Delegación del Gobierno de Madrid. Estadísticas sobre delitos en la ciudad de Madrid. Madrid: Delgación del Gobierno de Madrid; 1998.

8. Soares LE, Sento Sé JT, DeSouza Rodriguez JA, Piquet L. Criminalidade urbana e violencia no contexto internacional. En: Soares LE. Violência e política no Rio de Janeiro. Rio de Janeiro: ISER Relume Dumará; 1996. pp. 165-188.

9. Adorno S. La criminalidad violenta en Brasil: tendencias y características. Rev Ven Econ Ciencias Sociales 1997;3(2-3): 163-181. 
10. Roberts D. Mortalidad por lesiones no intencionales y violencia en las Américas: libro de referencia. Washington, DC: Organización Panamericana de la Salud; 1997.

11. Cruz JM, González LA. Magnitud de la violencia en El Salvador. Estudios Centroamericanos ECA 1997; 52:953-966.

12. Carabineros de Chile. Estadísticas de delitos. Santiago: Carabineros de Chile; 1998

13. Organización Panamericana de la Salud. Desarrollo del plan de acción regional sobre violencia y salud. Washington, D.C.: OPS; 1996. (Documento mimeografiado.)

14. Guerra de Macedo C. Sociedad, violencia y salud. [Editorial.] Bol Oficina Sanit Panam 1994; 117(5):i.

15. Martín-Baró I. Acción e ideología: psicología social desde Centroamérica. 8a ed. San Salvador: UCA Editores; 1997.

16. Organización Panamericana de la Salud, División de Promoción y Protección de la Salud. Salud y violencia: plan de acción regional. Washington, D.C.: OPS; 1994.

17. Guerra de Macedo C. Sociedad, violencia y salud. Una nueva agenda para la democracia. En: Organización Panamericana de la Salud. Sociedad, violencia y salud. Memorias de la Conferencia Interamericana sobre Sociedad, Violencia y Salud. Washington, D.C.: Washington, DC: OPS; 1996: 9-16.

18. Organización Panamericana de la Salud. La violencia: un problema de salud pública que se agrava en la región. Bol Epidemiol 1990; 11:1-7.

19. Organización Panamericana de la Salud, División de Salud y Desarrollo Humano. Actitudes y normas culturales sobre la violencia en ciudades seleccionadas de la región de las Américas. Proyecto ACTIVA. Washington, D.C.: OPS; 1996. (Documento mimeografiado.)

20. Carrión F. De la violencia urbana a la convivencia ciudadana. En: Concha Eastman A, Carrión F, Cobo G, eds. Ciudad y violencias en América Latina. Quito: Programa de Gestión Urbana; 1994. pp. 5-22.

21. Vanderschueren F. La violencia urbana, los pobres de la ciudad y la justicia. En: Concha Eastman A, Carrión F, Cobo G, eds. Ciudad y violencias en América Latina. Quito: Programa de Gestión Urbana; 1994. pp. 5-22.

22. Schmalleger F. Criminology today. Englewood Cliffs: Prentice Hall; 1996.

23. Hentig $\mathrm{HH}$. Robo con fuerza en las cosas. Robo con violencia o intimidación. En: Hentig HH. Estudios de psicología criminal. 4a ed, Vol I. Madrid: Espasa-Calpe; 1980.

24. National Research Council. Understanding and preventing violence. Washington, DC National Academy Press; 1993.

25. Levine FJ, Rosich KJ. Social causes of violence: crafting a science agenda. Washington, D.C. American Sociological Asociation; 1996.

26. U.S. Department of Justice, Bureau of Justice Statistics. Criminal victimization in the United States, 1994. Washington, DC: U.S. Department of Justice; 1997. p. viii.

27. Yunes J. Mortalidad por causas violentas en la región de las Américas. Bol Oficina Sanit Panam 1993;114:302-314

28. Centro de Pesquisa e Documentação de História Contemporânea do Brasil, Instituto de Estudos da Religião. Lei, justiça e cidadania. Direito, victimização e cultura política na região metropolitana do Rio de Janeiro. Rio de Janeiro: CPDOC.FGV/ISER; 1997.

29. Concha A, et al. Estrategias de la alcaldía de Cali para enfrentar la inseguridad y la violencia. En: Concha Eastman A, Carrión F, Cobo $\mathrm{G}$, eds. Ciudad y violencias en América Latina. Quito: Programa de Gestión Urbana; 1994. pp. 121-153.

30. Cruz JM. Los factores posibilitadores y las expresiones de la violencia en los noventa. Estudios Centroam ECA 1997;52:977-991.

31. Yunes J, Rajs D. Tendência de la mortalidade por causas violentas en la población general e entre los adolescentes y jóvenes de la região de las Américas 1994. Cad Saude Publica 1994;10(supl. 1):88-125.

32. Akerman M. Mapa de risco da violência da cidade de São Paulo: explorando os diferenciais intra-urbanos. Presentado en Seminario sobre violencia criminal urbana. Rio de Janeiro: Banco Interamericano de Desarrollo; 1997. (Documento mimeografiado.)

33. Oviedo Saavedra ER. Percepción de inseguridad en la ciudad. Entre lo imaginario y lo real. El caso del gran Santiago. En: Concha Eastman A, Carrión F, Cobo G, eds. Ciudad y violencias en América Latina. Quito: Programa de Gestión Urbana; 1994. pp. 275-312.

34. Guerrero R. Prevención de la violencia a través del control de sus factores de riesgo. Presentado en Reunión sobre El Desafío de la Violencia Criminal Urbana. Rio de Janeiro: Banco Interamericano de Desarrollo; 1997. (Documento mimeografiado.)

35. Sisti E. Consideraciones preliminares sobre la investigación de la violencia como problema de salud pública, a través de puntos centinelas. San Salvador: Instituto Universitario de Opinión Pública; 1997. (Documento mimeografiado.)

36. Organización Panamericana de la Salud, Coordinación de Investigaciones. Proyecto ACTIVA. Base de datos ACTIVA. Washington, D.C.: OPS, 1999. (Serie Investigaciones en Salud Pública, Documento Técnico No. 5).

37. Ferrán Aranaz M. SPSS para Windows. Programación y análisis estadístico. Madrid: McGraw Hill; 1996.

38. Hopkins KD, Hopkins BR, Glass GV. Estadística básica para las ciencias sociales y del comportamiento. México: Prentice-Hall Hispanoamericana; 1997.

39. Siegel S. Estadística no paramétrica aplicada a las ciencias de la conducta. México, D.F.: Editorial Trillas; 1974.
ABSTRACT

Being a victim of urban violence: its likelihood and its associated variables in cities of Latin America and Spain
This article looks at eight cities in Latin America and Spain and compares the chances of and variables associated with being a victim of various kinds of urban violence. The analysis was done using data from the ACTIVA Multicenter Study, which was coordinated by the Pan American Health Organization in 1996. The study sample consisted of 10821 persons who were representative of residents between the ages of 18 and 70 in eight metropolitan areas of Latin America and Spain: Rio de Janeiro, Brazil; Salvador, Brazil; Santiago, Chile; Cali, Colombia; San José, Costa Rica; San Salvador, El Salvador; Caracas, Venezuela; and Madrid, Spain. The results show that the likelihood of being a victim of different types of violence varies from city to city. The variables associated with a greater likelihood of being a victim in most - though not allof the cities are sex, age, and consumption of alcohol. 